\title{
Effects of adjuvant radiotherapy on borderline and malignant phyllodes tumors: A systematic review and meta-analysis
}

\author{
SHIYAN ZENG ${ }^{1}$, XINDAN ZHANG $^{2}$, DEJUAN YANG ${ }^{1}$, XIAOYI WANG ${ }^{1}$ and GUOSHENG REN ${ }^{1}$ \\ Departments of ${ }^{1}$ Breast and Endocrine Surgery; ${ }^{2}$ Cardiology, The First Affiliated Hospital \\ of Chongqing Medical University, Chongqing 400016, P.R. China
}

Received September 10, 2014; Accepted December 19, 2014

DOI: $10.3892 / \operatorname{mco} .2015 .503$

\begin{abstract}
The standard treatment for borderline and malignant phyllodes tumors is wide local excision (margins $\geq 1 \mathrm{~cm}$ ), in the context of either breast-conserving surgery (BCS) or total mastectomy (TM). Due to the high risk of local recurrence (LR) following surgical intervention alone, the addition of adjuvant radiotherapy (RT) has been previously investigated; however, the conclusions have been inconsistent. This systematic review and meta-analysis was designed to assess the efficacy of adjuvant RT for borderline and malignant phyllodes tumors. PubMed and Web of Science were systematically searched to identify relevant studies assessing the effect of adjuvant RT on borderline and malignant phyllodes tumors from the inception of this technique through May, 2014. A total of 8 studies were identified among 332 citations. In this meta-analysis, patients who received adjuvant RT had a lower relative risk of LR [hazard ratio $(\mathrm{HR})=0.43,95 \%$ confidence interval (CI): 0.23-0.64]. The absolute risk difference was $10.1 \%$ (95\% CI: 4.9-17.6), corresponding to a number needed to treat of 10 . Our pooled meta-analysis clearly demonstrated a decreased risk of LR in patients with borderline and malignant phyllodes tumors who received RT following BCS (HR=0.31, 95\% CI: -0.10-0.72). However, the combined HR for LR in the TM group did not demonstrate that adjuvant RT was superior to no RT (HR=0.68, 95\% CI: -0.28-1.64). No significant differences were observed in overall survival (OS) or disease-free survival (DFS) between the two groups. Our analysis suggested that adjuvant RT for borderline and malignant phyllodes tumors decreased the LR rate in patients undergoing BCS. However, adjuvant RT was not found to exert an effect on OS or DFS.
\end{abstract}

Correspondence to: Professor Guosheng Ren, Department of Breast and Endocrine Surgery, The First Affiliated Hospital of Chongqing Medical University, 1 You Yi Road, Yuanjiagang, Yuzhong, Chongqing 400016, P.R. China

E-mail:rgs726@163.com

Key words: phyllodes tumors, radiotherapy, local recurrence, overall survival, disease-free survival

\section{Introduction}

Phyllodes tumors have a low incidence ( 1 in 100,000 women) and account for only $0.5 \%$ of all breast neoplasms (1). The majority of these tumors arise in women aged 35-55 years (approximately 20 years later compared to fibroadenomas) (2). In men, phyllodes tumors are very rare, with only few cases described to date $(3,4)$. The World Health Organisation classifies phyllodes tumors into benign, borderline and malignant, according to the histopathological characteristics. Borderline and malignant phyllodes tumors are distinguished from benign phyllodes tumors by the presence of 'moderate or marked stromal cellularity and atypia, stromal overgrowth, brisk mitotic activity ( $\geq 5 / 10$ high-power fields) and permeative margins' (5). Clinically, borderline and malignant phyllodes tumors are characterized by their propensity for local and distant recurrence. The primary treatment for borderline and malignant phyllodes tumors is wide local excision (margins $\geq 1 \mathrm{~cm}$ ), in the context of either breast-conserving surgery (BCS) or total mastectomy (TM) (1,6-18). Local recurrence (LR) occurs in 10-65\% of the patients and distant recurrence rates range between 5 and $40 \%$ postoperatively $(7,8,11,12,19-24)$. Due to the high risk of LR following surgical intervention alone, the addition of adjuvant radiotherapy (RT) to the treatment of borderline and malignant phyllodes tumors has been previously investigated; however, the conclusions have been inconsistent. Furthermore, an observational study reported a trend toward increased utilization of RT, despite its uncertain effect on outcome (25). The current guidelines only recommend consideration of RT for malignant phyllodes in the setting of LR (level 2B evidence) (26).

We thus performed a systematic review and meta-analysis to assess the efficacy of adjuvant RT for borderline and malignant phyllodes tumors.

\section{Materials and methods}

Study selection. The focus of this systematic review and meta-analysis was to specifically assess the postoperative outcomes of borderline and malignant phyllodes tumors with and without RT. A systematic search of PubMed and Web of Science was undertaken, using the following terms: (phyllodes tumor OR phyllodes tumour OR phyllodes tumors OR phyllodes tumours OR cystosarcoma phyllodes OR cystosarcoma phyllode OR phyllode tumor OR phyllode tumour OR phyllode tumors 
OR phyllode tumours OR biphasic tumors OR biphasic tumor OR biphasic tumour OR biphasic tumours OR cystosarcoma phylloides OR cystosarcoma phylloides tumor OR cystosarcoma phylloides tumors OR cystosarcoma phylloides tumour OR cystosarcoma phylloides tumours OR phylloides tumor OR phylloides tumors OR phylloides tumour OR phylloides tumours) and (breast OR mammary OR mammory OR mammary glands OR mammary gland OR mammory gland OR mammory glands) and (radiation therapy OR radiotherapy OR radiation oncology OR radiation $O R$ radiation treatment $O R$ radiotreatment $O R$ ray therapeutics OR ray treatment), up to May 1, 2014.

Inclusion and exclusion criteria. The inclusion criteria were as follows: The studies had to be primary research articles specifically investigating the outcomes of postoperative borderline and malignant phyllodes tumors with and without RT; at least one subgroup analysis comprising borderline or malignant phyllodes tumors was to be reported. Patients with positive margins were excluded. To increase the number of potentially interesting articles, no limits or language restriction were applied during the search.

Reviews and case reports on the subject were excluded. Published abstracts without complete articles were excluded due to our inability to obtain detailed information. All the citations were independently reviewed by two authors (SYZ and XDZ) and categorized as relevant, not relevant, or potentially relevant. Citations that were classified as relevant and potentially relevant by one of the authors were selected for abstract review. Following review of the abstract, potentially relevant and relevant abstracts were selected for full-text evaluation. Upon evaluation of the full text, patients were included if they were surgically treated for borderline or malignant phyllodes tumor and information on the use of RT was available; women with history of breast cancer or distant metastatic disease at presentation were excluded.

Extracted data. The following information was extracted from each eligible study: Authors' names, source of patients, number of patients, patient age, type of surgery, margin status, radiation dose, follow-up period and conclusion (Table I). The numbers of events were extracted to conduct a meta-analysis of the different outcomes under investigation. If the numbers of events were not available, the LR rates or survival rates were used to estimate them.

Statistical analysis. Stata software, version 12.0 (StataCorp, College Station, Texas, USA) was used to pool the different outcome estimates. The outcomes were analyzed as hazard ratios (HRs). Statistical heterogeneity was assessed using the $\mathrm{I}^{2}$ statistic (27). We considered $\mathrm{I}^{2}<25 \%$ to represent low heterogeneity, $\mathrm{I}^{2}>75 \%$ high heterogeneity and $25 \%<\mathrm{I}^{2}<75 \%$ moderate heterogeneity. The evidence classification was performed using GRADEprofiler software, version 3.6 (the Cochrane Collaboration Network). We classed outcomes as high-, moderate-, low- or very low-quality $(28,29)$.

Next to relative outcome measures, we calculated pooled absolute risks (ARs). The AR difference was calculated as the pooled estimate of the AR difference for each study, including a 95\% confidence interval (CI). Subsequently, the number needed to treat was calculated as 1 divided by the AR difference.

\section{Results}

Study selection process. A total of 332 unique citations were identified following a search through PubMed and Web of Science after removing duplicates by EndNote; of the 332 articles, 87 were selected for abstract review and, of those, full-text evaluation was undertaken for 23 publications (Fig. 1). Overall, 6 publications were excluded due to lack of sufficient data; 4 were excluded as they were reviews; 3 were excluded as they did not focus on the outcome under investigation and 2 were excluded due to overlapping. Finally, a total of 8 studies were included in the present systematic review and meta-analysis $(13,14,25,30-34)$.

Description of studies. The characteristics of the included studies are listed in Table I. The total number of participants included in this systematic review and meta-analysis was 2,708. The studies were published between 2001 and 2014 . Inclusion in all the studies was restricted to patients with primary borderline and malignant phyllodes tumors, without identified metastasis at presentation. All the patients underwent surgery and the majority $(\sim 90 \%)$ had negative resection margins. A proportion of the patients consented to undergo RT. None of the patients had a history of breast cancer or other malignant tumors and none had priorly received adjuvant RT. In the studies, only a small proportion of the patients $(<1 \%)$ also received chemotherapy or endocrine therapy during the treatment. Therefore, we decided to include all these patients in the meta-analysis.

Moreover, the primary outcome of the majority of the studies was LR, which was defined as recurrence or a secondary phyllodes tumor in the ipsilateral breast. Frequent secondary outcomes were overall survival (OS) and disease-free survival (DFS). Since our meta-analysis only included 8 studies, we did not perform formal tests for funnel plot asymmetry.

Meta-analysis. Of the 8 studies included in the meta-analysis, 6 studies described LR. Since Gnerlich et al (25) reported the HR of LR and did not report the exact number of LR in patients with and without RT, we calculated the 5-year risk ratio (RR) of the remaining 5 involved studies. The pooled analysis confirmed a lower relative risk of LR in patients who received RT ( $R R=0.45$, 95\% CI: 0.26-0.77). Subsequently, a pooled analysis of these 6 studies also demonstrated a lower relative risk of $\mathrm{LR}$ in patients with $\mathrm{RT}(\mathrm{HR}=0.43$, 95\% CI: 0.23-0.64) with an $\mathrm{I}^{2}$ of $0 \%$, indicating low heterogeneity (Fig. 2). A meta-analysis on the different types of surgery is likely to be very helpful in this regard, identifying subgroups of patients undergoing BCS and TM. The meta-analysis was repeated without the study results of Soumarova et al (31) and Pandey et al (32), as there was no available information for comparable subgroups (BCS vs. TM). The pooled HR of LR in the BCS group demonstrated a lower relative risk of LR in patients with RT compared to those not receiving RT (HR=0.31, 95\% CI: -0.10-0.72); however, the combined HR for LR in the TM group did not indicate that adjuvant RT was superior to no RT (HR=0.68, 95\% CI: -0.28-1.64; Fig. 3).

Of the included studies, 4 reported data on OS. The results of our pooled meta-analysis demonstrated that surgery plus RT vs. surgery alone was associated with a decreased 


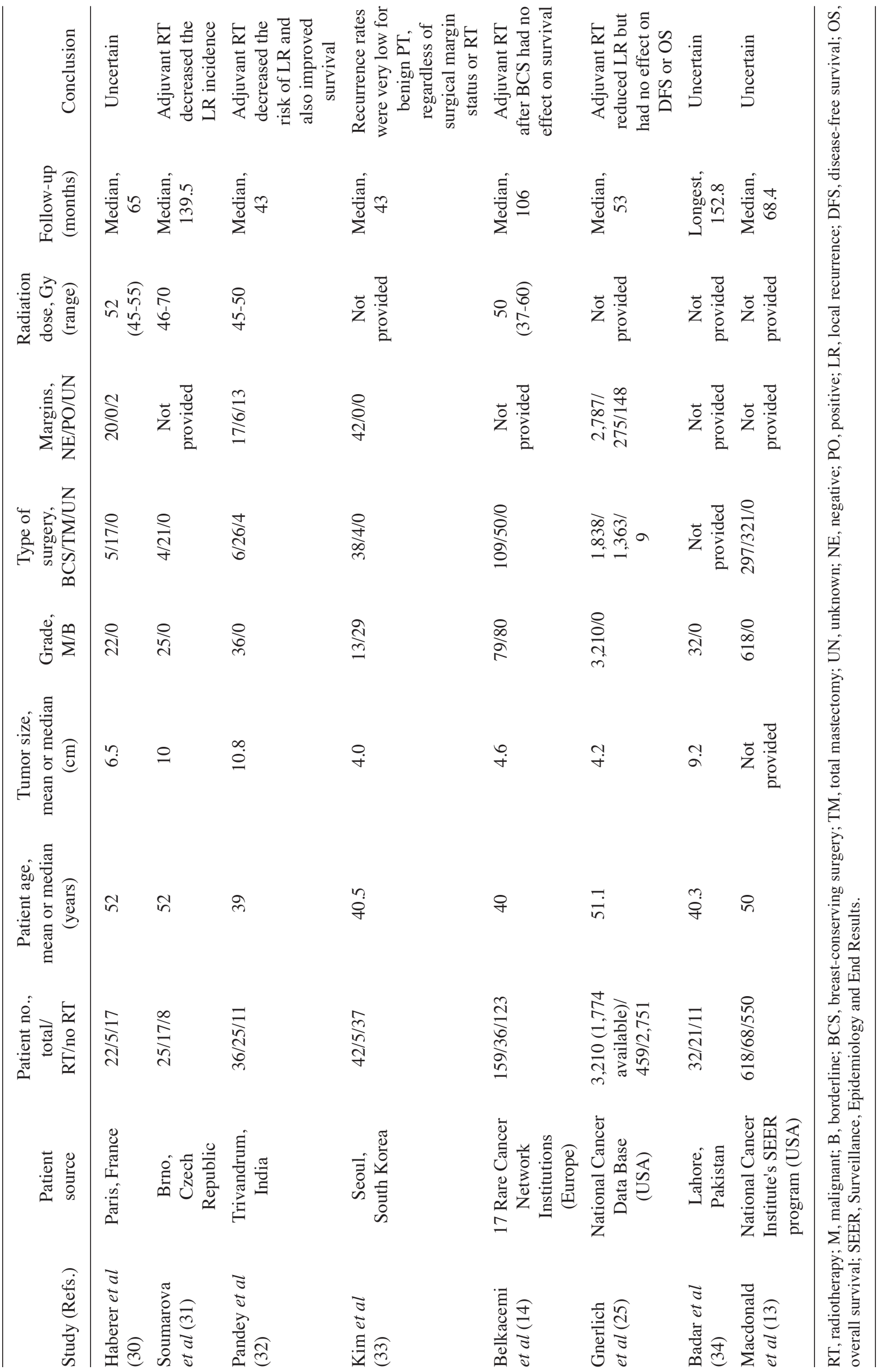




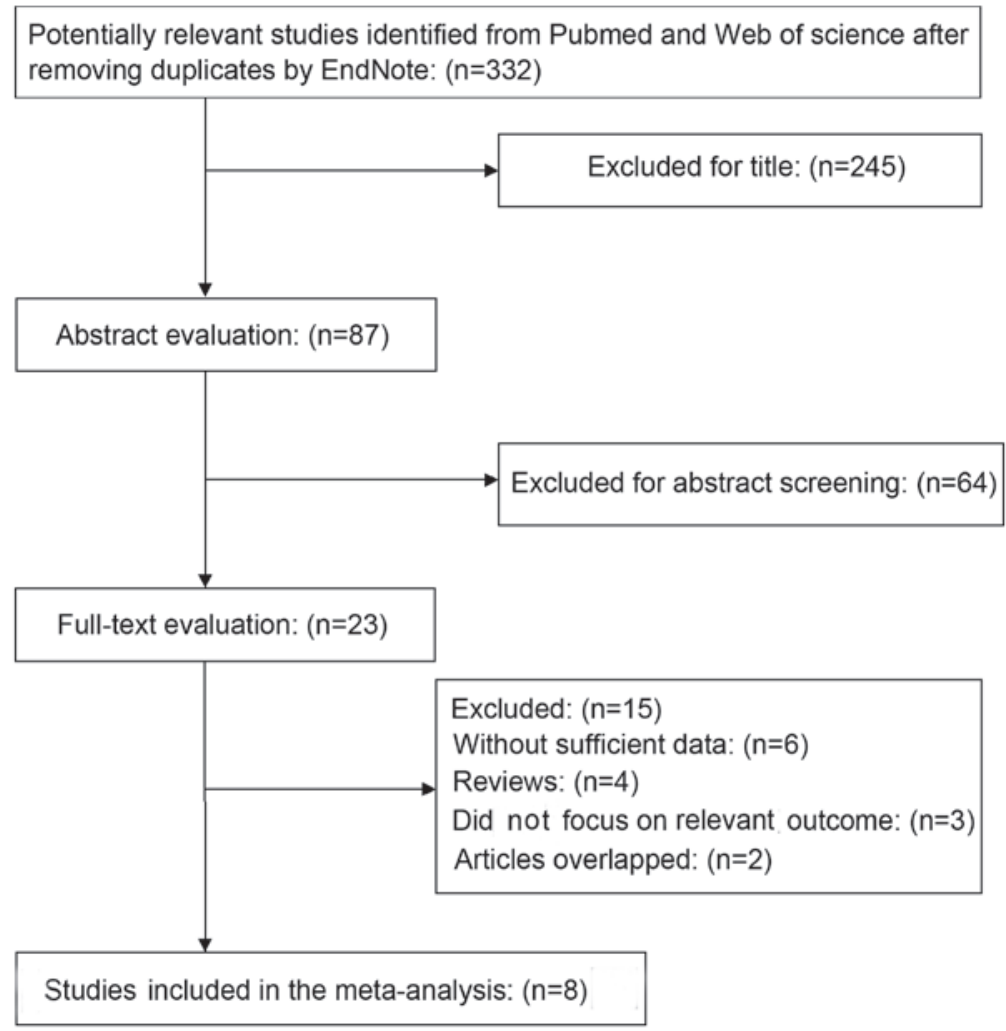

Figure 1. Results of the literature search and disposition of articles screened for inclusion.

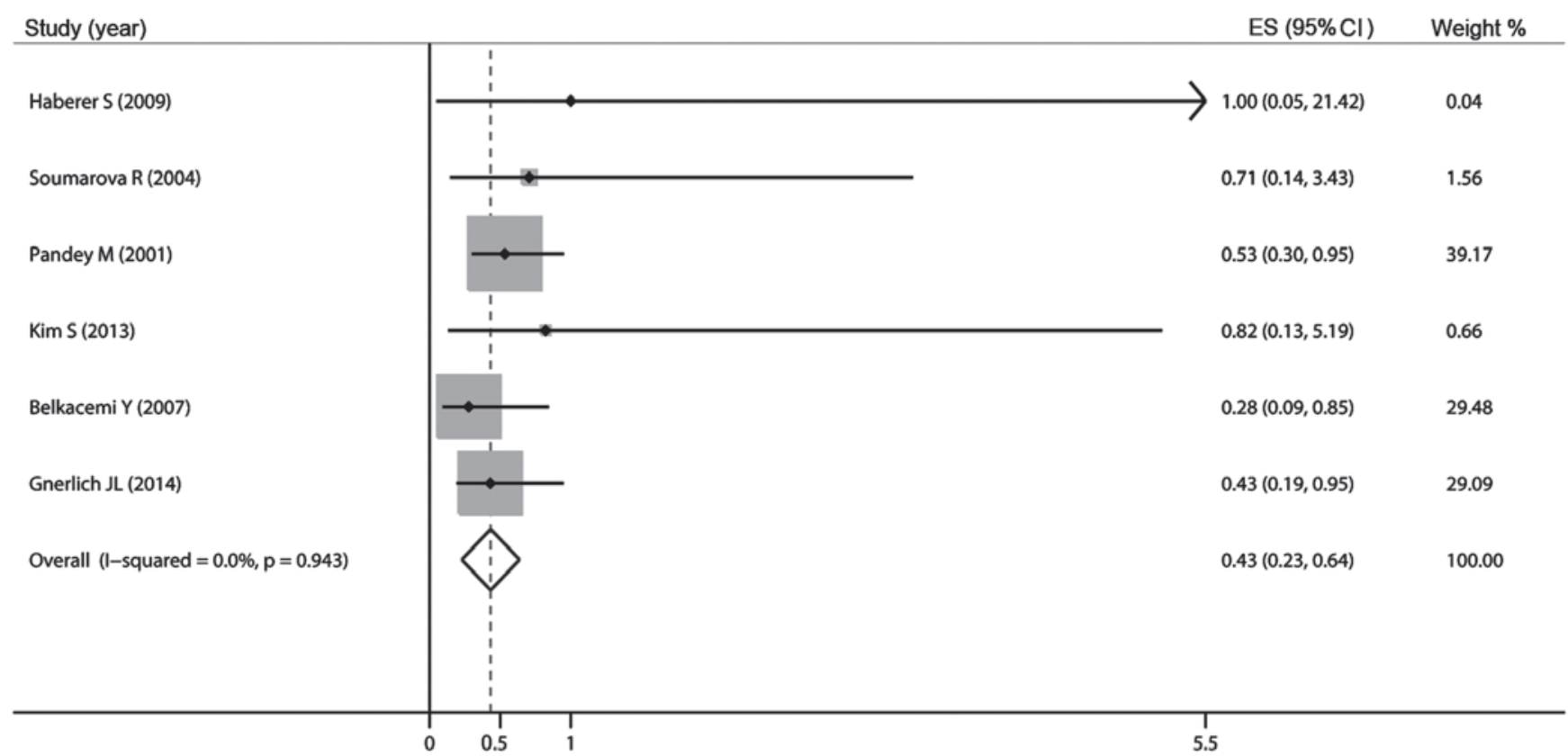

Figure 2. Pooled results of local recurrence in patients treated with adjuvant radiotherapy. ES, effect size; CI, confidence interval.

5-year OS (HR=0.89, 95\% CI: 0.79-0.99; Fig. 4) with an $\mathrm{I}^{2}$ of $58.0 \%$, indicating moderate heterogeneity. The meta-analysis was repeated without the study results of Badar et al (34), as there was no available information for comparable subgroups (BCS vs. TM). However, in the subgroups by different types of surgery, the pooled HR of OS in the BCS group $(\mathrm{HR}=0.95,95 \% \mathrm{CI}: 0.79-1.12)$ and the TM group $(\mathrm{HR}=0.89$,
95\% CI: 0.73-1.04; Fig. 5) revealed that adjuvant RT exerted no effect on 5-year OS.

A total of 4 studies reported on the effect of RT on DFS. The results of this pooled analysis demonstrated that adjuvant RT following surgery exerted no effect on 5-year DFS (HR=0.93, 95\% CI: 0.74-1.12; Fig. 6) and the $\mathrm{I}^{2}$ of DFS was $37.8 \%$, indicating moderate heterogeneity. A meta-analysis 


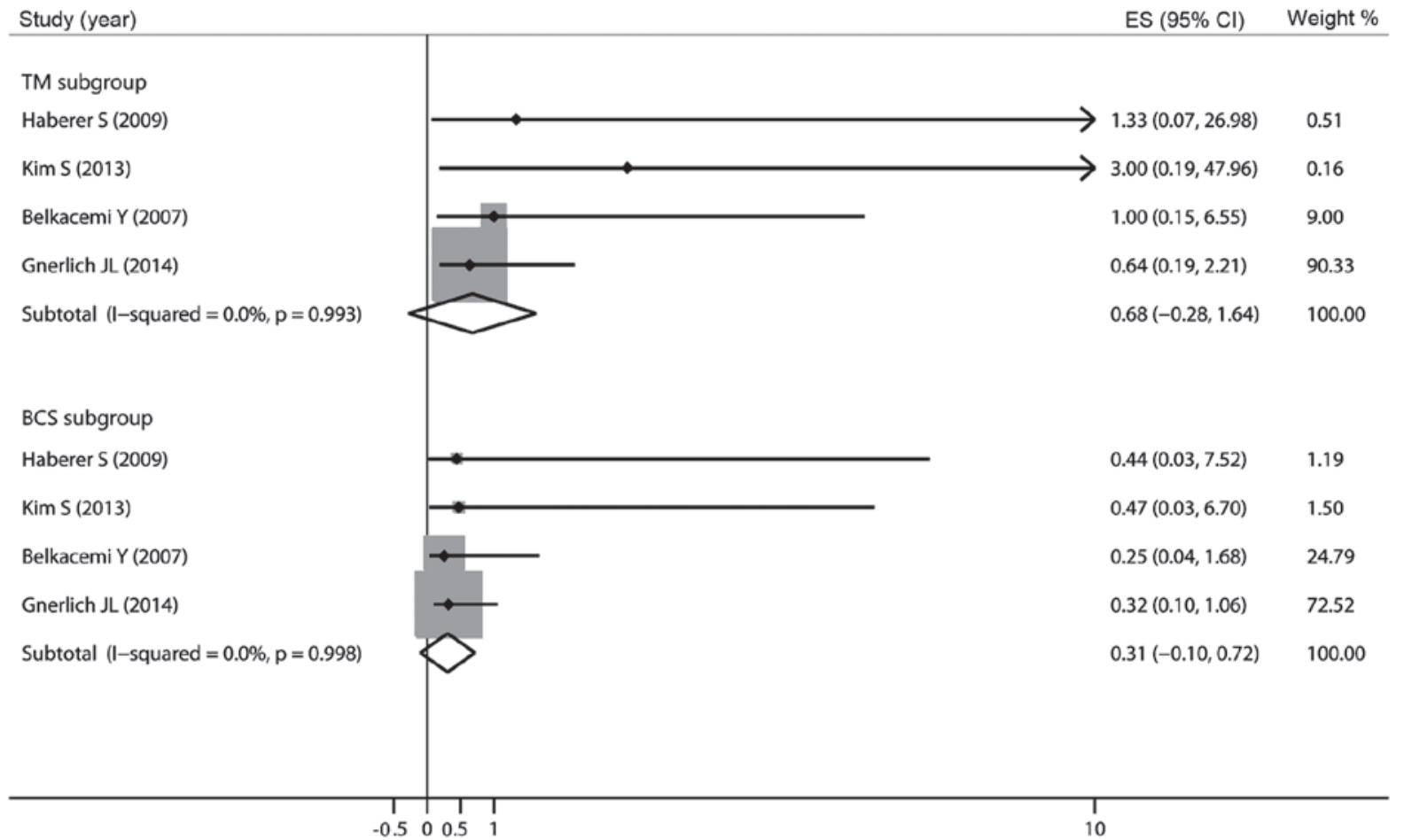

Figure 3. Pooled results of local recurrence in subgroups of patients treated with adjuvant radiotherapy. ES, effect size; CI, confidence interval.

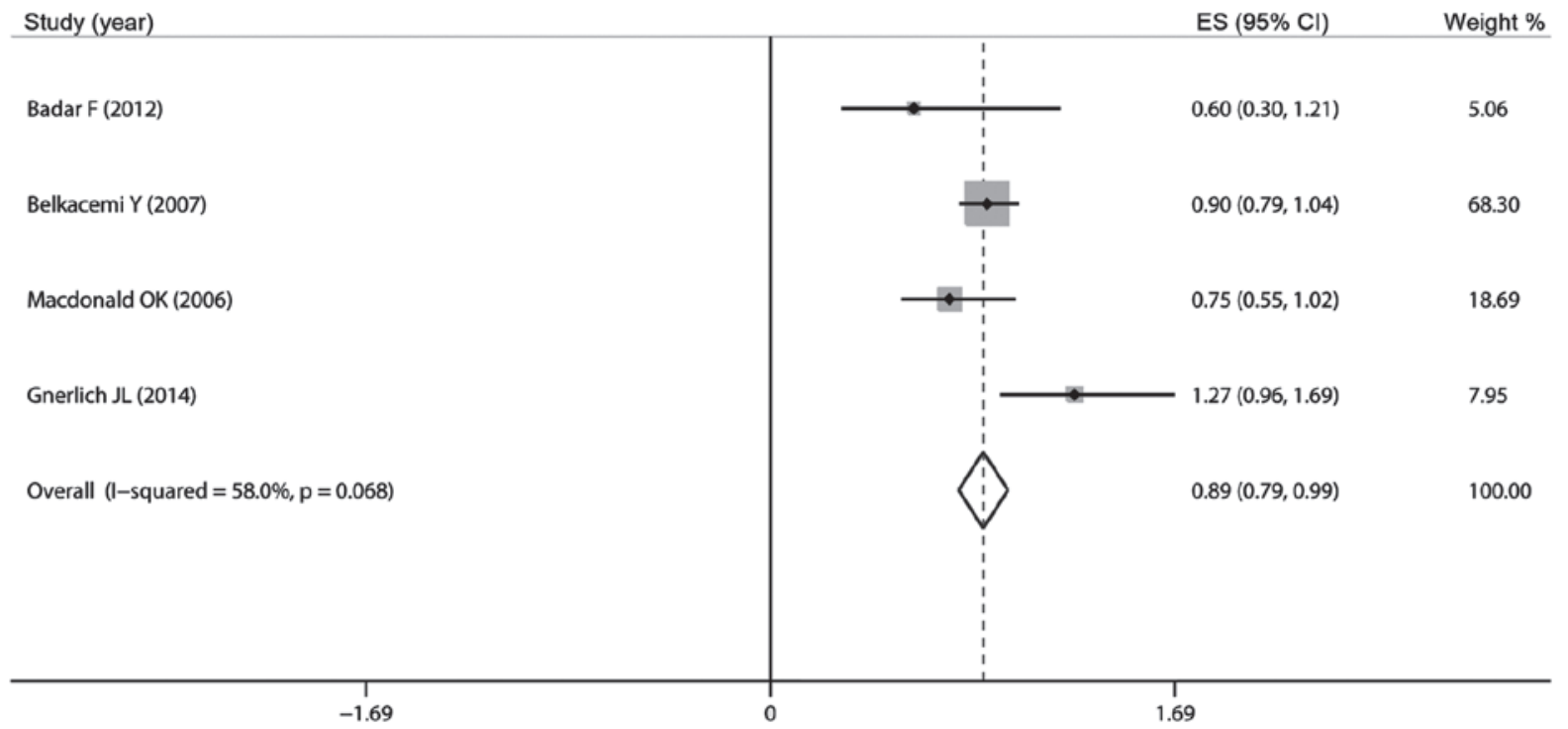

Figure 4. Pooled results of overall survival in patients treated with adjuvant radiotherapy. ES, effect size; CI, confidence interval.

of specific subgroups was not possible, as information from half of the studies were available for incomparable subgroups alone. Based on the Grading of Recommendations Assessment, Development and Evaluation (GRADE) system, the level of evidence of the involved studies was very low (Table II).

$A R$. The pooled AR of LR, OS and DFS were calculated for patients in both study arms. Since ARs are dependent on the duration of follow-up, we calculated the 5-year AR of these outcomes. The 5-year AR of a LR among patients who received RT was $19.3 \%$ (17 of $88,95 \%$ CI: $11.6-29.1$ ) compared to $29.4 \%$
(58 of 197, 95\% CI: 23.1-36.3) among patients who did not receive RT. The AR difference was 10.1\% (95\% CI: 4.9-17.6). The results were in favor of those who received RT in addition to surgery, corresponding to a number needed to treat of 10 to prevent one LR in 5 years.

The 5-year AR of OS was 52\% (65 of 125, 95\% CI: 42.9-61.0) in patients who received RT compared to $59.2 \%$ (405 of 684, 95\% CI: 55.4-62.9) in those who did not. For DFS, the 5-year ARs were 59.8\% (49 of 82, 95\% CI: 48.3-70.4) among patients who received RT compared to $63.9 \%$ (92 of 144, 95\% CI: 55.5-71.7) among those who did not. 


\begin{tabular}{|c|c|c|c|}
\hline Study (year) & & ES (95\% Cl) & Weight $\%$ \\
\hline TM subgroup & & & \\
\hline Belkacemi Y (2007) & $\rightarrow-$ & $0.95(0.76,1.19)$ & 53.99 \\
\hline Macdonald OK (2006) & $\rightarrow$ & $0.65(0.42,1.00)$ & 30.34 \\
\hline Gnerlich JL (2014) & $\longrightarrow$ & $1.11(0.78,1.58)$ & 15.67 \\
\hline Subtotal $(I-$ squared $=53.2 \%, p=0.118)$ & $\diamond$ & $0.89(0.73,1.04)$ & 100.00 \\
\hline BCS subgroup & & & \\
\hline Belkacemi Y (2007) & & $0.94(0.77,1.13)$ & 81.68 \\
\hline Macdonald OK (2006) & $\longrightarrow$ & $0.90(0.57,1.41)$ & 14.96 \\
\hline Gnerlich JL (2014) & & $1.63(0.97,2.74)$ & 3.36 \\
\hline Subtotal $(I-$ squared $=14.7 \%, p=0.310)$ & & $0.95(0.79,1.12)$ & 100.00 \\
\hline
\end{tabular}

Figure 5. Pooled results of overall survival in subgroups of patients treated with adjuvant radiotherapy. ES, effect size; CI, confidence interval.

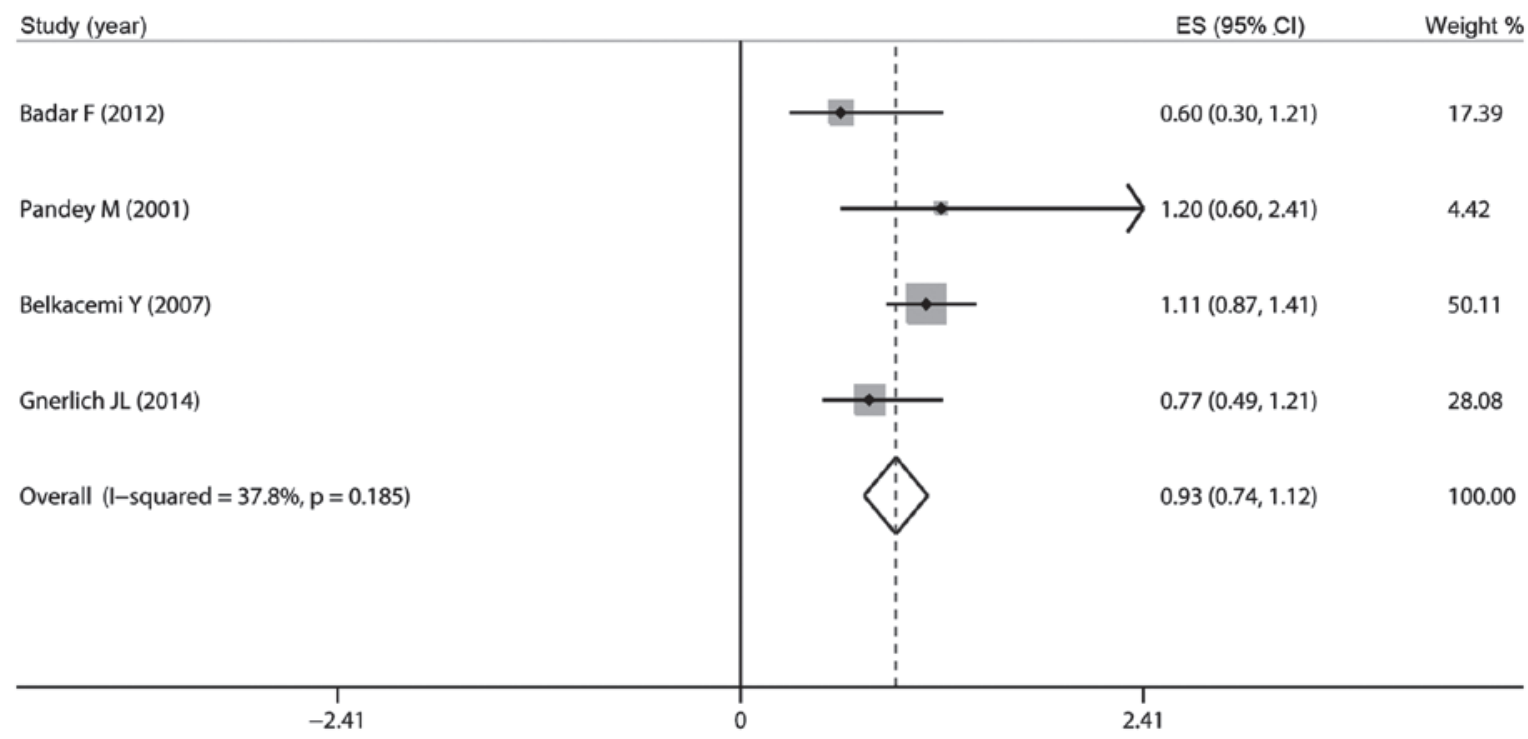

Figure 6. Pooled results of disease-free survival in patients treated with adjuvant radiotherapy. ES, effect size; CI, confidence interval.

\section{Discussion}

To the best of our knowledge, this is the first systematic review and meta-analysis to investigate the effects of adjuvant RT on borderline and malignant phyllodes tumors. Our pooled meta-analysis clearly demonstrated a decreased risk of LR in patients with borderline and malignant phyllodes tumors who received RT following BCS. The results of this pooled analysis revealed that adjuvant RT exerts no effect on OS or DFS.

The standard treatment for borderline and malignant phyllodes tumors is wide local excision (margins $\geq 1 \mathrm{~cm}$ ), in the context of either BCS or TM. However, the risk of LR following margin-negative resection of borderline or malignant phyllodes tumors is significant. Although the addition of adjuvant RT to the treatment of borderline and malignant phyllodes tumors has been investigated, no consensus has been reached. Gnerlich et al (25) reported a significant increase in
RT use for BCS as well as TM patients, despite its uncertain efficacy. Therefore, we performed this systematic review and meta-analysis to integrate the results from recent studies that investigated the effect of adjuvant RT on borderline and malignant phyllodes tumors.

Our pooled analysis confirmed a lower relative risk of LR in patients receiving adjuvant RT following surgical resection compared to surgery alone. The AR difference was $10.1 \%$ (95\% CI: 4.9-17.6). The results were in favor of patients who received RT in addition to surgery, corresponding to a number needed to treat of 10 to prevent one LR in 5 years. This number is expected to be relatively higher in a non-trial population. To decrease the number needed to treat and to personalize treatment, we attempted to identify subgroups of patients (BCS vs. TM) in which RT may be safely omitted on the basis of the risk of LR. The pooled HR of LR in the BCS group revealed a lower relative risk of LR (HR=0.31, 95\% CI: $-0.10-0.72$ ); 


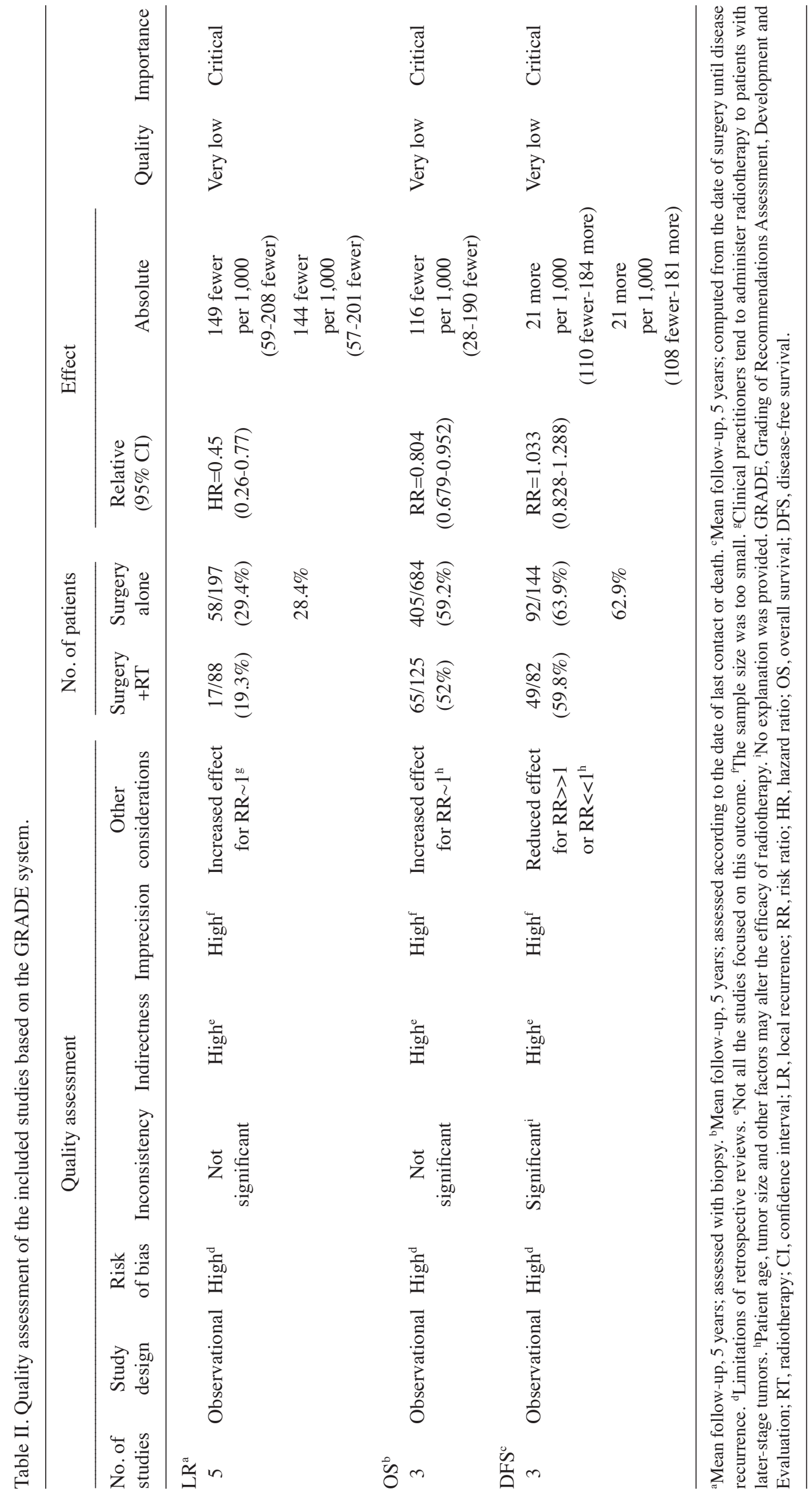


however, the combined HR for LR in the TM group did not demonstrate that adjuvant RT was superior to no $\mathrm{RT}(\mathrm{HR}=0.68$, 95\% CI: -0.28-1.64). Thus, omission of RT in patients following TM may be reasonable. However, these results must be interpreted with caution, considering that we only calculated the 5-year LR rate in TM patients. Belkacémi et al (14) observed similar 5-year local control rates in irradiated patients following TM compared to those who did not receive RT (92 vs. $91 \%$, respectively); however, adjuvant RT improved the 10-year local control for borderline and malignant tumors (92 vs. 78\%, respectively). Thus, following TM, adjuvant RT should be discussed taking into account the surgical margins, size and pathological criteria of the tumor. Pezner et al (22) recommend adjuvant RT following TM for large tumors $(>10 \mathrm{~cm})$.

Our results were consistent with those of the individual studies. We also identified 1 study that reported marginally different results. Reinfuss et al (35) reported on patients who were treated with the Halsted operation followed by postoperative RT, which proved to be inefficient. In their study, patients who received postoperative irradiation exhibited infiltration of the pectoralis major muscle and limited mobility of the chest wall; in addition, the surgical margin was not microscopically tumor-free. After 5 years of follow-up, all the patients exhibited LRs along with pulmonary metastases. Only few of the patients involved in our study exhibited infiltration of the pectoralis major muscle and the majority of the patients $(\sim 90 \%)$ had negative resection margins. The difference between their results and ours may be explained by the worse overall condition of the patients in the study of Reinfuss et al (35). The study of Barth et al (20) yielded similar conclusions to ours. In their prospective study, 46 women (30 with malignant and 16 with borderline phyllodes tumors) were treated with margin-negative breast-conserving resection followed by adjuvant RT. The patients were treated in 30 different institutions; after a minimum follow-up of 5 years, none of the patients exhibited LR. A retrospective study by Chaney et al (36) that involved 6 patients treated with adjuvant RT for non-metastatic phyllodes tumor of the breast yielded similar results. There was no reported local or distant failure after a median follow-up of 36.5 months. Therefore, adjuvant RT should be considered to minimize LRs following breast-conserving resection of borderline and malignant phyllodes tumors.

To our surprise, our pooled meta-analysis demonstrated that surgery plus RT was associated with decreased 5-year OS compared to surgery alone ( $\mathrm{HR}=0.89,95 \% \mathrm{CI}$ : 0.79-0.99). To the best of our knowledge, there is no study reporting that postoperative RT decreases OS. None of our included individual studies indicated that adjuvant RT exerted a detrimental effect on OS. Moreover, the study by Pandey et al (32) demonstrated that adjuvant RT not only decreased the risk of LR, but also improved survival. We performed a subgroup analysis by exclusion of the study by Badar et al (34), as it lacks information on comparable subgroups (BCS vs. TM). The pooled HRs of OS in the BCS group (HR=0.95, 95\% CI: 0.79-1.12) and the TM group $(\mathrm{HR}=0.89,95 \% \mathrm{CI}: 0.73-1.04)$ revealed that adjuvant $\mathrm{RT}$ exerted no effect on OS. This may be due to the small sample size in each group. Furthermore, RT is often administered to patients of poor prognosis. The wide CI may be due to variations in the survival data. Additional randomized controlled studies (RCTs) are required to further elucidate this issue.
Our pooled analysis proved that RT following surgery exerted no effect on 5-year DFS. The 4 included individual studies did not report that adjuvant RT exerted a detrimental effect on DFS. Thus far, we have not identified any studies reporting different results. The 5-year AR of LR among patients who received RT was $19.3 \%$ (17 of 88 , 95\% CI: $11.6-29.1$ ), vs. $29.4 \%$ (58 of $197,95 \%$ CI: $23.1-36.3$ ) among patients who did not receive $\mathrm{RT}$. The data of Kim et al (33) and Barth et al (20) demonstrated that the AR of LR following margin-negative breast-conserving resection of borderline and malignant phyllodes tumors was 16/73 (22\%) and 30/134 (22\%), respectively. However, our pooled AR data were higher. This may be explained as follows: Although we tried to eliminate patients with positive resection margins, such patients could not be eliminated in 2 of the included studies, whereas the other 2 included studies did not provide data on margin status. An estimated $10 \%$ of those patients had positive margins. In this case, our study indicates that adjuvant RT may be effective for patients with positive tumor resection margins.

The goal of adjuvant RT is to reduce disease recurrence and improve the quality of life of the patients. From the time adjuvant RT was first introduced as part of breast cancer treatment, this procedure has gained in popularity and it has been shown to reduce disease recurrence and improve the quality of life. A number of studies have supported the oncological effectiveness and safety of this procedure in phyllodes tumors $(20,32,36)$. Certain clinical practitioners remain cautious regarding adjuvant RT in phyllodes tumors. Our analysis supports the conclusion that postoperative RT for borderline and malignant phyllodes tumors decreases the $\mathrm{LR}$ rate for patients undergoing BCS. However, adjuvant RT is not the only factor determining whether patients will develop a relapse; other factors, including age, surgical approach, mitotic activity and surgical margins are significantly correlated with recurrence $(\mathrm{P}=0.029,0.020,0.048$ and 0.00018 , respectively) (15). Local excision, wide excision, or TM with negative surgical margins yielded high local control rates, but local excision was associated with a relatively high percentage of positive surgical margins (18.3\%) (15). Pezner et al (22) retrospectively analysed 478 patients with malignant phyllodes tumors of the breast and found that the 5-year local control rates were $100 \%$ for $0-2-\mathrm{cm}$ tumors, $95 \%$ for $2-5-\mathrm{cm}$ tumors, $88 \%$ for $5-10-\mathrm{cm}$ tumors and $85 \%$ for $10-20-\mathrm{cm}$ tumors. A multivariate analysis of OS found several factors to be significant, including advancing age with each decade after 50 years, appearance of distant metastases, larger tumor size and local control vs. LR $(\mathrm{HR}=2.5, \mathrm{P}<0.05)(22)$. Surgical management must be tailored to the clinical situation, with more aggressive management reserved for higher-grade or recurrent tumors (19). In the latter case, if wide local excision is possible while allowing satisfactory cosmesis, BCS could also be considered. Pezner et al (22) recommend adjuvant RT following BCS for tumor sizes $>2 \mathrm{~cm}$.

There were certain limitations to this analysis. First, we did not consider certain confounding factors, such as chemotherapy and endocrine therapy, as the original data were unavailable, although these patients accounted for an estimated $<1 \%$. However, these factors may affect the rate of recurrence. Second, selection bias, particularly the tendency to administer adjuvant RT to patients with later-stage tumors, 
was a problem in the majority of the studies. Third, the different follow-up duration may also limit the interpretation of the results. Fourth, all the studies in our analysis were non-RCTs, the results of which are not as convincing as those of RCTs. Finally, according to the GRADE system, the level of the included studies' evidence was very low. Therefore, further investigation may alter our conclusions.

In conclusion, this meta-analysis suggests that adjuvant RT for borderline and malignant phyllodes tumors decreases the LR rate for patients undergoing BCS. However, adjuvant RT exerts no effect on OS or DFS. The majority of the studies were performed via retrospective analysis to investigate small numbers of patients. Therefore, we believe that a multicenter prospective RCT with a longer follow-up period and more clearly defined parameters may be the best way to further elucidate this issue in the future.

\section{References}

1. Moffat CJ, Pinder SE, Dixon AR, Elston CW, Blamey RW and Ellis IO: Phyllodes tumours of the breast: a clinicopathological review of thirty-two cases. Histopathology 27: 205-218, 1995.

2. Bernstein L, Deapen D and Ross RK: The descriptive epidemiology of malignant cystosarcoma phyllodes tumors of the breast. Cancer 71: 3020-3024, 1993

3. Bartoli C, Zurrida SM and Clemente C: Phyllodes tumor in a male patient with bilateral gynaecomastia induced by oestrogen therapy for prostatic carcinoma. Eur J Surg Oncol 17: 215-217, 1991.

4. Keelan PA, Myers JL, Wold LE, Katzmann JA and Gibney DJ: Phyllodes tumor: clinicopathologic review of 60 patients and flow cytometric analysis in 30 patients. Hum Pathol 23: 1048-1054, 1992.

5. Tan PH, Jayabaskar T, Chuah KL, et al: Phyllodes tumors of the breast: the role of pathologic parameters. Am J Clin Pathol 123 529-540, 2005

6. Rowell MD, Perry RR, Hsiu JG and Barranco SC: Phyllodes tumors. Am J Surg 165: 376-379, 1993.

7. Reinfuss M, Mitus J, Duda K, Stelmach A, Rys J and Smolak K: The treatment and prognosis of patients with phyllodes tumor of the breast: an analysis of 170 cases. Cancer 77: 910-916, 1996.

8. Barth RJ and Jr: Histologic features predict local recurrence after breast conserving therapy of phyllodes tumors. Breast Cancer Res Treat 57: 291-295, 1999.

9. Tan PH, Thike AA, Tan WJ, et al; Phyllodes Tumour Network Singapore: Predicting clinical behaviour of breast phyllodes tumours: a nomogram based on histological criteria and surgical margins. J Clin Pathol 65: 69-76, 2012.

10. Abdalla HM and Sakr MA: Predictive factors of local recurrence and survival following primary surgical treatment of phyllodes tumors of the breast. J Egypt Natl Canc Inst 18: 125-133, 2006.

11. Chaney AW, Pollack A, McNeese MD, et al: Primary treatment of cystosarcoma phyllodes of the breast. Cancer 89: 1502-1511, 2000

12. Asoglu O, Ugurlu MM, Blanchard K, et al: Risk factors for recurrence and death after primary surgical treatment of malignant phyllodes tumors. Ann Surg Oncol 11: 1011-1017, 2004.

13. Macdonald OK, Lee CM, Tward JD, Chappel CD and Gaffney DK: Malignant phyllodes tumor of the female breast: association of primary therapy with cause-specific survival from the Surveillance, Epidemiology, and End Results (SEER) program. Cancer 107: 2127-2133, 2006.

14. Belkacémi Y, Bousquet G, Marsiglia $\mathrm{H}$, et al: Phyllodes tumor of the breast. Int J Radiat Oncol Biol Phys 70: 492-500, 2008.
15. Chen WH, Cheng SP, Tzen CY, et al: Surgical treatment of phyllodes tumors of the breast: retrospective review of 172 cases. J Surg Oncol 91: 185-194, 2005.

16. Mangi AA, Smith BL, Gadd MA, Tanabe KK, Ott MJ and Souba WW: Surgical management of phyllodes tumors. Arch Surg 134: 487-492, 1999.

17. Kapiris I, Nasiri N, A'Hern R, Healy V and Gui GP: Outcome and predictive factors of local recurrence and distant metastases following primary surgical treatment of high-grade malignant phyllodes tumours of the breast. Eur J Surg Oncol 27: 723-730, 2001.

18. Barrio AV, Clark BD, Goldberg JI, et al: Clinicopathologic features and long-term outcomes of 293 phyllodes tumors of the breast. Ann Surg Oncol 14: 2961-2970, 2007.

19. Guillot E, Couturaud B, Reyal F, et al: Management of phyllodes breast tumors. Breast J 17: 129-137, 2011.

20. Barth RJ Jr, Wells WA, Mitchell SE and Cole BF: A prospective, multi-institutional study of adjuvant radiotherapy after resection of malignant phyllodes tumors. Ann Surg Oncol 16: 2288-2294, 2009.

21. Ellis I, Sawyer EJ, Rampaul R and Pineda CG: Phyllodes tumor of the breast. In: Textbook of Uncommon Cancer. Raghavan D, Brecher ML, Johnson DH, Meropol NJ, Moots PL and Rose PG (eds). 3rd edition. Wiley, Chichester: pp209-217, 2006.

22. Pezner RD, Schultheiss TE and Paz IB: Malignant phyllodes tumor of the breast: local control rates with surgery alone. Int J Radiat Oncol Biol Phys 71: 710-713, 2008.

23. Parker SJ and Harries SA: Phyllodes tumours. Postgrad Med J 77: 428-435, 2001

24. J Barth R Jr: Margin negative, breast conserving resection: adequate for benign phyllodes tumors, but inadequate therapy for borderline and malignant phyllodes tumors. Breast Cancer Res Treat 142: 463-464, 2013.

25. Gnerlich JL, Williams RT, Yao K, Jaskowiak N and Kulkarni SA: Utilization of radiotherapy for malignant phyllodes tumors: analysis of the National Cancer Data Base, 1998-2009. Ann Surg Oncol 21: 1222-1230, 2014.

26. Carlson RW, Allred DC, Anderson BO, et al: Metastatic breast cancer, version 1.2012: featured updates to the NCCN guidelines. J Natl Compr Canc Netw 10: 821-829, 2012.

27. Higgins JP and Thompson SG: Quantifying heterogeneity in a meta-analysis. Stat Med 21: 1539-1558, 2002.

28. Atkins D, Best D, Briss PA, et al; GRADE Working Group: Grading quality of evidence and strength of recommendations. BMJ 328: 1490, 2004

29. Jaeschke R, Guyatt GH, Dellinger P, et al: Use of GRADE grid to reach decisions on clinical practice guidelines when consensus is elusive. BMJ 337: a744, 2008 (In French).

30. Haberer S, Laé M, Seegers V, et al: Management of malignant phyllodes tumors of the breast: the experience of the Institut Curie. Cancer Radiother 13: 305-312, 2009.

31. SoumarováR,SeneklováZ,HorováH,etal: Retrospective analysis of 25 women with malignant cystosarcoma phyllodes - treatment results. Arch Gynecol Obstet 269: 278-281, 2004.

32. Pandey M, Mathew A, Kattoor J, et al: Malignant phyllodes tumor. Breast J 7: 411-416, 2001.

33. Kim S, Kim JY, Kim do H, Jung WH and Koo JS: Analysis of phyllodes tumor recurrence according to the histologic grade. Breast Cancer Res Treat 141: 353-363, 2013.

34. Badar F, Mahmood S, Syed AA and Siddiqui N: Malignant phyllodes tumour of the breast. J Ayub Med Coll Abbottabad 24: 47-49, 2012

35. Reinfuss M, Mituś J, Smolak K and Stelmach A: Malignant phyllodes tumours of the breast. A clinical and pathological analysis of 55 cases. Eur J Cancer 29A: 1252-1256, 1993.

36. Chaney AW, Pollack A, McNeese MD and Zagars GK: Adjuvant radiotherapy for phyllodes tumor of breast. Radiat Oncol Investig 6: 264-267, 1998 